\title{
On-line Digital-darkfield TEM Determination of Nanocrystal 3D-lattices
}

\author{
P Fraundorf ${ }^{1}$ \\ 1. Physics \& Astronomy Dept. \& Center for Nanoscience, U. Missouri-Saint Louis, MO, USA \\ 2. Physics Department, Washington University, Saint Louis, MO, USA
}

Given a field of view in the TEM that contains many crystals, it has long been possible to form a darkfield image of each diffraction spot, noting which particle lights up, and to then tilt to new orientations and repeat the process. By after the fact combining information on tilt and diffraction spot locations, the full 3D reciprocal lattice (or a non-trivial subset thereof) can then be determined for all crystals for which one has data on 3 or more non-coplanar diffraction spots, as can the oriented basis triplet for that crystal should one desire to send the beam down a specified lattice direction in that crystal [1]. Each spot beyond the first 3 also provides information on lattice parameter uncertainties.

The same thing can be done using crystallographic data in lattice images [2-4], by simply taking images of a polycrystalline field at several orientations and performing the same analysis using digital darkfield techniques [5-7] after the fact. Now let's imagine that your microscope is integrated with a computer which can in real time analyze darkfield data in lattice images or diffraction, and moreover record and control goniometer stage orientations at the same time. Computer support in such cases should allow one to select a field of nanocrystals, and ask the microscope to determine the 3D lattice parameters of each along with their absolute (and relative) lattice orientations.

We don't yet have a microscope that can do this, but we have built an on-line simulator in JS/HTML5 that can do "strong phase-object" (single-slice) digital darkfield imaging of polycrystalline specimens [8], and in the days ahead will let us prototype user interfaces for microscopes that can do such analysis with real specimens. The simulator already assists one in measuring the spacings and angles between periodicity pairs seen in a single specimen orientation.

Subsequent objectives are to add an ability to report spacings and angles between periodicity pairs flagged in arbitrary specimen orientations. On the analysis side, the first step (now done on-line in separate JS code) is to examine options for indexing any measured spacing/angle set against a candidate lattice. The next step is to record an arbitrary collection of selected periodicities, selected by mouse clicks, followed by an attempt to come up with a 3D reciprocal lattice from scratch able to span the full set.

If the operator has selected periodicities associated with a single crystal, then within error of the selection process the routine should be able to not just "better weed out candidates", but to determine the 3D lattice parameters themselves. Determining the "conventional cell choice" will still require some guesses, since e.g. a 90 degree angle and a 91 degree angle may say very different things about which space group is involved.

This is only the beginning of course, as the selection of periodicities is still being left up to the operator. However it is a robust start, and suggestions for making such an interface better are invited. Our next steps beyond that with the on-line simulator likely will shift focus back to nanoeducation, with options to zoom in/out and translate for "electron exploration" of procedurally generated nanoworlds. 


\section{References:}

[1] P. Fraundorf, Ultramicroscopy 6 (1981), p. 227.

[2] P. Fraundorf, Ultramicroscopy 22 (1987), p. 225.

[3] Wentao Qin, Haresh Siriwardane and Phil Fraundorf, Microscopy \& Microanalysis 5(S2) (1999).

[4] Wentao Qin and P. Fraundorf, Ultramicroscopy 94(3-4) (2003), p. 246.

[5] P. Fraundorf and G. K. Fraundorf, Proc. $47^{\text {th }}$ Annual Meeting of the Electron Microscope Society of America (1989), p. 122.

[6] P. Fraundorf and Lu Fei, Microscopy and MicroAnalysis 10 (S2), (2004), p. 300.

[7] P. Fraundorf and Chris Bishop, Microscopy and MicroAnalysis 19(S2) (2013), p. 1804.

[8] P. Fraundorf et al, Microscopy and Microanalysis 22(S3) (2016), p. 1442.
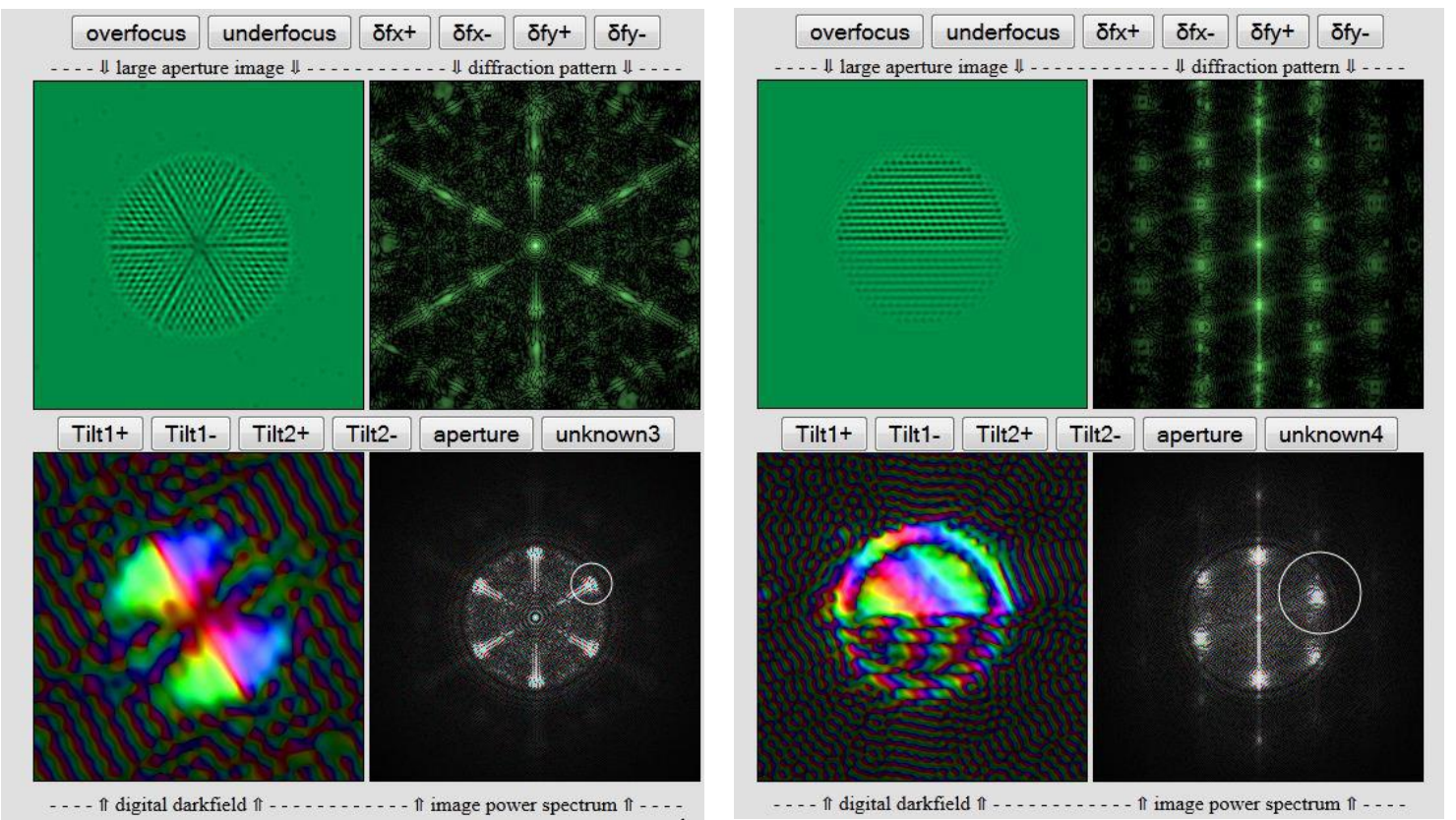

Figure 1. (left): Digital darkfield image (bottom left panel) of an icosahedral twin, with a pair of crystals sharing a common $\{111\}$ plane lit up in our on-line simulator in our on-line JS/HTML5 simulator at sites.google.com/site/electrondetectives.

Figure 2. (right): Digital darkfield image (bottom left panel) of an $\mathrm{Au} / \mathrm{ZnO}$ sphere with the $\mathrm{Au}$ hemisphere associated with the circled spot in the image power spectrum (bottom right panel), also in that strong-phase object (single-slice) simulator. 